\title{
L'évacuation et l'utilisation rationnelles des ordures ménagères; tâche importante de notre temps, et la nécessité d'une planitication adéquate des recherches pertinentes par la coopération internationale
}

\author{
Par O. JAAG \\ Directeur de l'Institut fédéral pour l'aménagcment, l'épuration \\ et la protection des eaux, E.P.F., Zurich \\ Manuscrit reçu le 30 août I95s
}

Dans de nombreux pays européens, sinon dans tous les pays de ce continent, l'évacuation des ordures ménagères est devenue récemment non seulement une tâche actuelle, mais aussi une tâche urgente, dans le cadre même de la propreté des villes, grandes ou petites. Dans notre pays tout au moins, les temps sont révolus où les ordures solides des cuisines, ménages et jardins étaient simplement déposées au bord des lacs ou des fleuves, dans des vallons forestiers, ou en pleins champs. Le sens esthétique de l'homme se refuse à voir de beaux paysages enlaidis de cette manière comme on refuse également de nos jours de tolérer tous les désavantages que présente le dépôt d'ordures en plein air, notamment odeurs répugnantes, infestations d'insectes et de rats, danger d'incendies, etc.

En outre, on a constaté récemment que ces dépôts d'ordures ont eu une influence désastreuse sur l'équilibre chimique et biologique de nombreux lacs et rivières comme aussi sur leurs conditions hygiéniques et que même les eaux souterraines ont été polluées au point de devenir inutilisables, ces eaux qui constituent une réserve si précieuse d'eau potable et autre pour les villes, les villages, l'industrie et l'agriculture. Au cours de la session printanière, notre parlement fédéral a adopté à l'unanimité et sans aucune abstention une loi fédérale sur la protection des eaux de surface et des eaux souterraines. Cette loi, qui est sur le point d'entrer en vigueur, interdit expressément tout dépôt de corps solides susceptibles de polluer d'une 
manière quelconque, directement ou indirectement, des eaux de surface ou souterraines, courantes ou stagnantes.

Il existe des villes, je mentionnerai en Suisse et à titre d'exemple Zurich, Bâle, Lausanne et Genève, comme aussi des villages (Davos) qui ont résolu le problème de façon satisfaisante en incinérant les ordures ménagères. Plus récemment, des communes rurales, Kusnacht et Ruschlikon dans le canton de Zurich, La Chaux-de-Fonds dans le Jura, ont commencé à transformer les ordures ménagères en compost, par les procédés du triage, de la réduction ou de la putréfaction. Toutefois, il y a encore des centaines, même des milliers de communes, dans notre seul pays, qui se trouvent encore dans l'obligation de réexaminer une fois de plus leur problème des ordures ménagères. Toutes ces communes s'efforcent de trouver une solution à la situation sur la base des connaissances scientifiques acquises, des réalisations techniques et des expériences pratiques, de trouver la solution parfaite du point de vue hygiénique comme du point de vue économique.

Les installations d'évacuation d'ordures ménagères fonctionnant déjà en Suisse ou à l'étranger, et celles en construction ont souvent servi de modèle pour l'établissement des plans et des calculs, elles ont en outre pesé dans les décisions. Les autorités municipales savent parfaitement qu'une installation qui leur est apparue excellente quelque part dans le monde ne saurait être adoptée telle quelle, mais qu'il faut au contraire l'adapter aux conditions particulières de leur propre collectivité.

Dans le choix d'une installation, il faut tenir compte tout d'abord de la grandeur de la commune, c'est-à-dire du nombre d'habitants dont les ordures ménagères doivent être évacuées. Alors que dans certains cas l'incinération des ordures est tout indiquée - dans les grandes villes ou dans les localités de moyenne importance notamment-, dans de nombreux autres cas il faut avoir recours à la transformation et à l'utilisation des ordures ménagères; et tout spécialement là où il y a une forte demande de compost d'ordures ménagères, et où l'écoulement de ce compost paraît assuré pour une période prolongée. Ce n'est que lorsque ces conditions sont remplies que se pose la question du choix de l'installation de transformation, et ceci de cas en cas. En Suisse, l'évacuation parfaite et ordonnée des ordures ménagères est apparue comme problème urgent assez récemment. Dans d'autres pays en revanche, et notamment aux Pays-Bas et au Danemark, pays où la culture du sol est très développée, en France, en Allemagne, en Grande-Bretagne et en Belgique également, on a recours depuis longtemps déjà aux ordures ménagères transformées. Voilà pourquoi ces pays ont élaboré des systèmes et méthodes de transformation d'ordures 
dont plusieurs ont trouvé application dans notre pays. Ces méthodes nous fournissent des expériences précieuses, et si nous ne pouvons les prendre comme modèles absolus vu les divergences existant dans les différents pays, nous pouvons toutefois les prendre comme base pour trouver ensuite une solution à nos propres problèmes d'évacuation d'ordures ménagères.

Cela ne veut pas dire que tous les problèmes d'évacuation d'ordures ménagères soient résolus, même dans les pays sus-nommés, et notre tâche nous apparait d'autant plus complexe si nous pensons aux conditions de chez nous! La décision sera toujours orientée vers la pensée suivante: Comment est-il possible de satisfaire, dans le sens le plus large du terme, aux exigences essentielles de l'hygiène et de l'économie, dans chaque cas particulier, par l'application des possibilités administratives et techniques. Aux Pays-Bas, au Danemark, en Allemagne et ailleurs, la question des ordures ménagères s'est posée sous un angle différent. Il s'agissait moins de l'évacuation des ordures à proprement parler, que d'un besoin pressant, de la demande d'humus pour engrais. Il s'agissait en effet de préparer pour des cultures intensives des sols sablonneux, pauvres en humus, des dunes ou des terrains de landes acides. Chez nous, les conditions sont différentes, car les terres réellement pauvres en humus ou acides sont plutôt rares.

Vu les conditions si variées dans les différents pays, il ne faut donc pas s'étonner que le problème de la transformation des ordures ménagères en engrais et produits d'amélioration des sols soit envisagé avec un intérêt plus ou moins grand, et que la valeur même du compost soit estimée de manière si différente. Lors de discussions d'experts à ce sujet, il se peut fort bien que l'un d'eux nie d'une façon générale le besoin de compost d'ordures ménagères, alors qu'un autre affirme et confirme ce besoin sans aucune restriction et s'étonne même qu'il y ait encore lieu de discuter d'une telle question.

Dans notre pays, il ne s'agit pas, dès l'abord de plaider la cause du compost d'ordures ménagères et de son emploi comme engrais, il s'agit au contraire d'examiner dans quels cas et pour quelles raisons économiques et agricoles il est possible d'utiliser le compost d'ordures ménagères.

$\mathrm{Si}$, lors de l'établissement du programme de notre symposium, nous avons accordé une place prédominante aux problèmes de la préparation des ordures ménagères pour les transformer en compost, cela ne veut pas dire que nous voudrions voir cette méthode se supplanter à l'incinération des ordures. L'incinération en effet a fait ses preuves et son importance sera maintenue, mais c'est une méthode d'évacuation qui ne recèle guère de problèmes essentiels à l'heure actuelle. 
Toutefois, dans le domaine de la transformation et de l'utilisation générale des ordures ménagères dans l'agriculture, il semble qu'il y ait des problèmes de base variés qui attendent une solution. Ces problèmes comprennent l'hygiène, les installations mécaniques, l'utilisation du compost d'ordures ménagères comme engrais et produit d'amélioration des sols, la valeur du compost de même que la comparaison entre les différentes méthodes de transformation du point de vue économique et la comparaison de ces méthodes avec le procédé d'incinération.

Il n'y aura jamais de formule unique quant à la méthode optimum d'évacuation des ordures ménagères. Les conditions varient en effet de pays à pays, de contrée à contrée, voire même de terrain à terrain dans une contrée donnée; il faut donc analyser tous les facteurs locaux et en tenir compte lors du choix de la méthode d'évacuation d'ordures ménagères.

Dans les cas où l'incinération des ordures ménagères est possible, c'està-dire là où le nombre d'habitants de la région est suffisant et où il n'y a pas de grande demande de compost, on aura avantage à donner la préférence à l'incinération et à utiliser la chaleur ainsi obtenue. Mais dans les très nombreux cas où ces conditions préalables n'existent pas, la transformation des ordures ménagères se justifiera. Or, ces cas sont très nombreux en Suisse et dans la plupart des autres pays où il y a des quantités de petites localités résidentielles et nous serions très heureux que les experts qui se trouvent parmi nous, experts en matière d'horticulture, de cultures maraîchères, de viniculture, de sylviculture et peut-être d'agriculture, veuillent bien nous mentionner un grand nombre de possibilités où le compost d'ordures ménagères peut être utilisé avec avantage.

Notre pays porte un intérêt particulier à ce problème de la transformation des ordures ménagères, là où il est possible de traiter conjointement les ordures et les boues des eaux usées, putréfiées ou fraîches.

Le décret suisse sur la livraison du lait qui est entré en vigueur à la fin de l'année dernière interdit désormais le ramassage de boue fermentée et son mélange au purin dans toutes les régions de production laitière. Une telle interdiction entraîne des difficultés catastrophales pour la protection des eaux. Elle exige en effet que les stations de purification des eaux usées construisent de plus grandes chambres de putréfaction, des compartiments de séchage de la boue plus vastes ou des installations mécaniques pour la condensation des boues, afin que ces matières puissent être entreposées et qu'elles soient prêtes au moment où l'on peut utiliser cette boue pour compost. Il faut donc que nous vouions une attention particulière à la transformation conjointe des boues et des ordures ménagères en compost, 
ce qui se fait déjà avec succès dans un certain nombre de pays, aux EtatsUnis, en Allemagne, au Danemark et en Angleterre notamment.

Quant aux discussions sur la valeur du compost d'ordures ménagères dans l'agriculture, prise dans son sens le plus large, elles révèlent toujours que des conceptions trop généralisées, des suppositions insuffisamment étayées orientent et déterminent en quelque sorte notre attitude envers ce problème, et qu'il y a encore de grandes lacunes à remplir dans les connaissances scientifiques et dans les expériences pratiques dont nous disposons dans ce domaine.

Avant de décider de la nécessité ou de l'opportunité de la transformation des ordures ménagères d'une collectivité, il y a lieu d'élucider un nombre important de problèmes partiels, sans quoi la solution du problème essentiel ne saurait être trouvée. Mais comme ces questions sont étudiées dans la quasi totalité des pays, sans qu'il y ait toutefois, dans bien des cas, les contacts et la coordination souhaitables avec les activités d'autres centres de recherche, il nous est apparu opportun d'examiner la situation actuelle au cours de discussions approfondies d'experts dans ce vaste domaine des ordures ménagères et d'essayer de donner à tous ceux qui s'occupent de recherches dans ce domaine un but un peu plus précis par une collaboration intelligente.

Les résultats obtenus dans les divers pays devraient être soumis aux experts d'autres pays de façon aussi complète et aussi rapidement que possible: Il faudrait en outre s'attaquer également aux questions qui n'ont pas reçu jusqu'à ce jour de réponse satisfaisante.

Voilà les considérations qui nous ont poussés, M. le directeur W. A. G. Weststrate, M. C. KaChulle, ingénieur, et moi-même, à vous inviter, vous, Messieurs les experts et vous, Messieurs les professionnels expérimentés, à discuter de ces questions. Nous vous remercions tous, vousmêmes et vos supérieurs, d'avoir accepté notre invitation avec tant d'empressement, et nous espérons que grâce à ces discussions qui se rapporteront aux problèmes choisis nous aurons une idée claire et nette qui nous aidera à diriger les recherches dans le domaine de la transformation et de l'utilisation des ordures ménagères vers une voie où elles seront couronnées de succès. 